\title{
LAS "SEÑORITAS DE ARAS", UN ELEMENTO GEOMORFOLOGICO SINGULAR AMENAZADO (PROVINCIA DE HUESCA, ESPAÑA)
}

\author{
Francisco PELLICER \\ Luis CANCER y \\ María Teresa ECHEVERRIA
}

Departamento de Geografía y Ordenación del Territorio

Universidad de Zaragoza

\begin{abstract}
Resumen: La construcción de una central hidroeléctrica en el barranco de Arás, afluente del río Gállego, provocaría la desaparición de un elemento morfológico singular, como es el de las "Señoritas de Arás".

El modelado de "dames coiffées" constituye un importante recurso geomorfológico, y por lo tanto paisajístico, de aquí que el conocimiento de su génesis y evolución sirva como un ejercicio educativo para la valoración del entorno natural y la evaluación de los impactos antrópicos.
\end{abstract}

Palabras clave: Modelado glacial, morrena, incisión fluvial, sistema morfoclimático, chimeneas de las hadas, impacto ambiental, paisaje.

Abstract: A the built of a power-station in the Aras Valley, a tributary of Gallego river, will destroy a unique morphological element: the "Señoritas de Aras".

The "dames Coiffées" landscape in an important geomorphological example, and the knowledge of its genesis and evolution serves as an educative exercise useful to get a good valoration of the natural environment and to evaluate the anthropic impacts.

Key words: Glacial ladnscape, moraine, fluvial down-culting, morphoclimatic system, "Dames Coiffées", hard impact, landscape. 


\section{INTRODUCCION}

Las "señoritas de Arás" son unas grandes columnas formadas en depósitos morrénicos con abundante matriz fina, coronadas por un bloque de material resistente que protege la base de la erosión. Estas llamativas formas reciben en la bibliografía geomorfológica el nombre de chimeneas de las hadas o dames coiffées o démoiselles coiffées. Las "señoritas de Arás" están localizadas en el barranco de Arás, afluente del río Gállego por la derecha, aguas abajo del núcleo de población de Biescas, en el Pirineo oscense.

Este trabajo pretende contemplar las "señoritas de Arás" como paisaje y como recurso natural. Ello significa introducir una nueva clave de comprensión, mirarlas desde la inteligencia y la sensibilidad, contemplar y descubrir el significado de sus aspectos geomorfológicos y estéticos e incluso de su utilidad más inmediata.

El paisaje de las "señoritas de Arás"puede contemplarse desde la razón que ordena, estructura y fundamenta para satisfacer la curiosidad que despiertan las caprichosas formas. El paisaje en su dimensión intelectual no se refiere solamente a la fisonomía o a los aspectos directamente perceptibles, sino que comprende también el criptosistema o información no visible sobre estructura y funcionamiento del sistema natural.

El paisaje tiene, asimismo, una dimensión sensitiva. El paisaje desde este punto de vista se identifica con la información que recibimos del entorno a través de los sentidos o fenosistema. Los estímulos emitidos por el paisaje, inicialmente objetivables, son distorsionados por el receptor de los mismos a través de filtros sensoriales, funcionales, culturales, afectivos y estéticos. Este tipo de estudios son escasamente abordados por los científicos a pesar de su inminente necesidad y utilidad.

Las chimeneas de las hadas son siempre morfologías singulares, singularidad que se presenta estrechamente ligada al grado de calidad medioambiental del paisaje y por lo tanto deben preservarse de los impactos que conlleven su degradación.

En la actualidad, y en relación con la dinámica remontante del barranco a lo largo del Cuaternario reciente, el grado de conservación de este modelado se encuentra en un estado crítico, siendo difícil predecir el período temporal que transcurriría, en condiciones normales, hasta su desmoronamiento definitivo. 


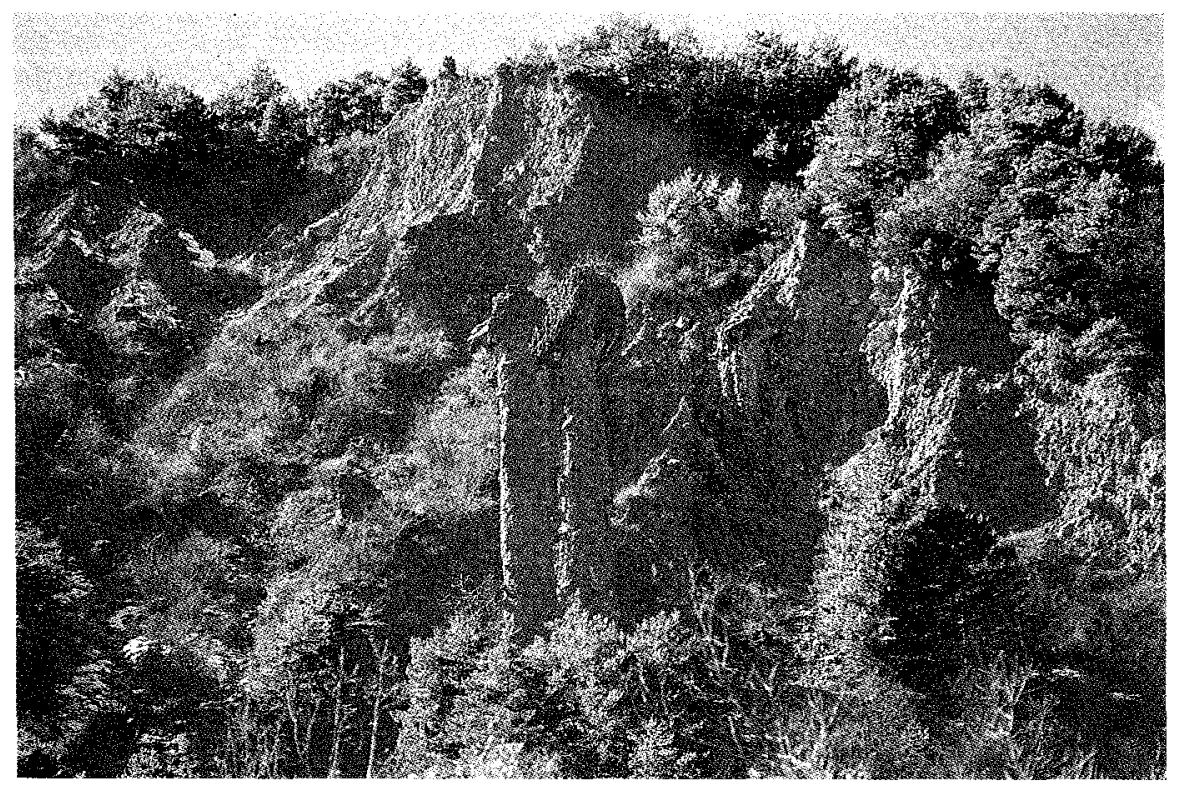

Fot. 1.- Vista general de las "Señoritas de Arás", modeladas sobre un depósito complejo de materiales morrénicos y de ladera.

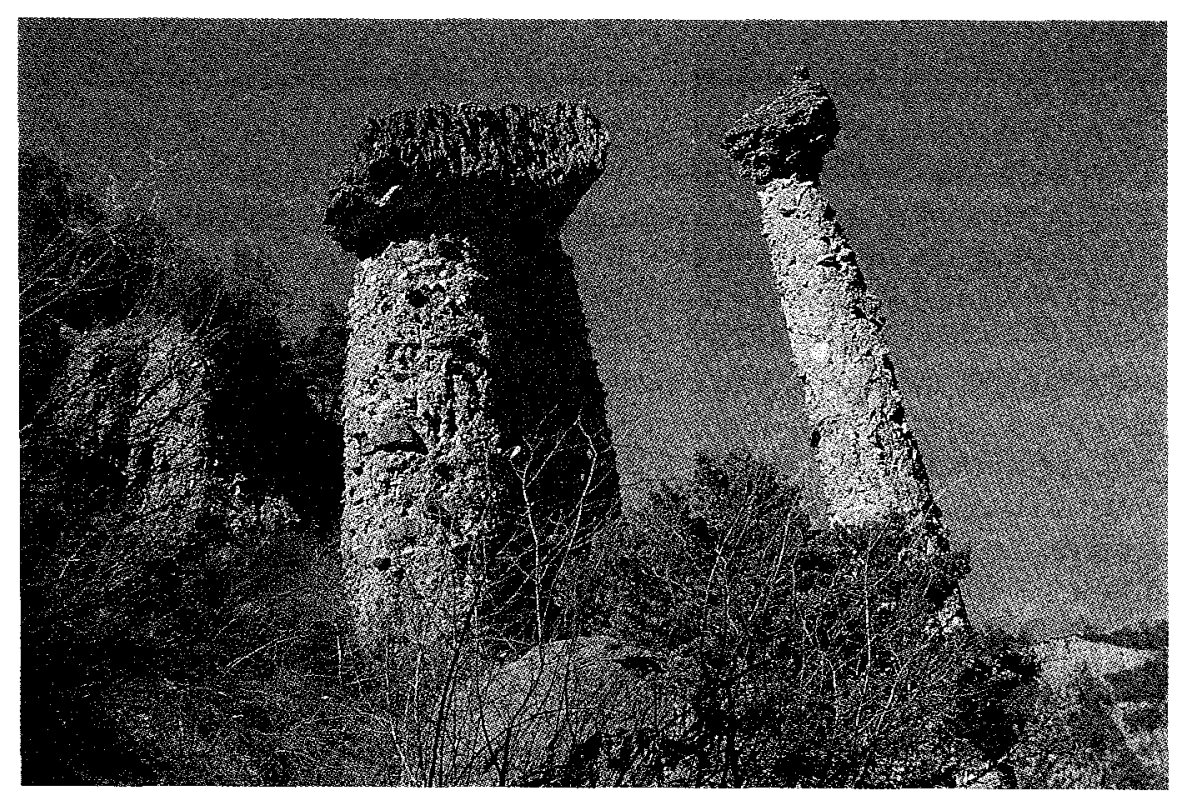

Fot. 2.- Detalle de las "Señoritas de Arás" que pone en evidencia su avanzado grado de evolución. 
Estas condiciones normales evolutivas, sin embargo, es posible que no lleguen a tener lugar. En este barranco se ha diseñado un proyecto de construcción de una "minicentral" hidroeléctrica, uno de cuyos elementos, el canal de derivación de las aguas transcurriría a muy pocos metros por encima de estas chimeneas. Este canal, de una longitud de aproximadamente $3000 \mathrm{~m}$., necesitaría para su ubicación una plataforma de $4 \mathrm{~m}$. de anchura. Tal y como reconocen los informes elaborados por la Diputación General de Aragón, las chimeneas desaparecerían con total seguridad por las simples vibraciones de la maquinaria, destruyéndose así una morfología de un gran valor ambiental, paisajístico y educativo.

\section{PRESENTACION DE LA ZONA DE ESTUDIO}

El Sobremonte, pequeña unidad espacial drenada por el barranco de Aso, se sitúa, tal y como ya se ha mencionado, en la cuenca del río Gállego, concretamente en la margen derecha del valle, aguas abajo de la población de Biescas.

El barranco de Aso nace cercano a los 2000 m., en las Peñas de Aso, y desemboca en el Gállego a unos $840 \mathrm{~m}$.; su cuenca presenta fuertes pendientes en los tramos superior e inferior, mientras que el valle medio del barranco de Aso se identifica con un rellano entre los 1200 y 1300 m., donde se sitúan las poblaciones de Aso, Yosa y Betés.

El sustrato geológico de la cuenca es el flysch eoceno, a caballo entre dos de las grandes unidades pirenaicas, las Sierras Interiores al Norte y la Depresión Media al Sur. El flysch pirenaico responde a una sedimentación en un medio de plataforma continental, integrada por secuencias carbonatadas, areniscas y margas, replegadas durante la orogenia alpina, presentando no obstante un sentido del desplome hacia el Sur. El carácter deleznable de las series y la inexistencia de potentes estratos resistentes han provocado un modelado indefinido de colinas y alineaciones de cumbres redondeadas y vertientes muy homogéneas, tal y como señalan GARCIA RUIZ y PUIGDEFABREGAS (1982), donde la evolución de laderas está relacionada con una acción fluvial de muy diversa magnitud, y con deslizamientos en masa. Estos mismo autores reconocen cierto escalonamiento altitudinal de los procesos de erosión, señalando que hasta los $1200 \mathrm{~m}$. las laderas están sometidas a procesos de arroyamiento difuso y "rigolas" de montaña media, y por encima de esta cota aumentan las acciones de erosión moderada y movimientos en masa de escasa entidad, localizándose frecuentes desprendimientos hasta los $1500 \mathrm{~m}$., y procesos solifluidales, arroyamiento superficial en surcos y cabeceras activas a más de 1600/1700 m. 


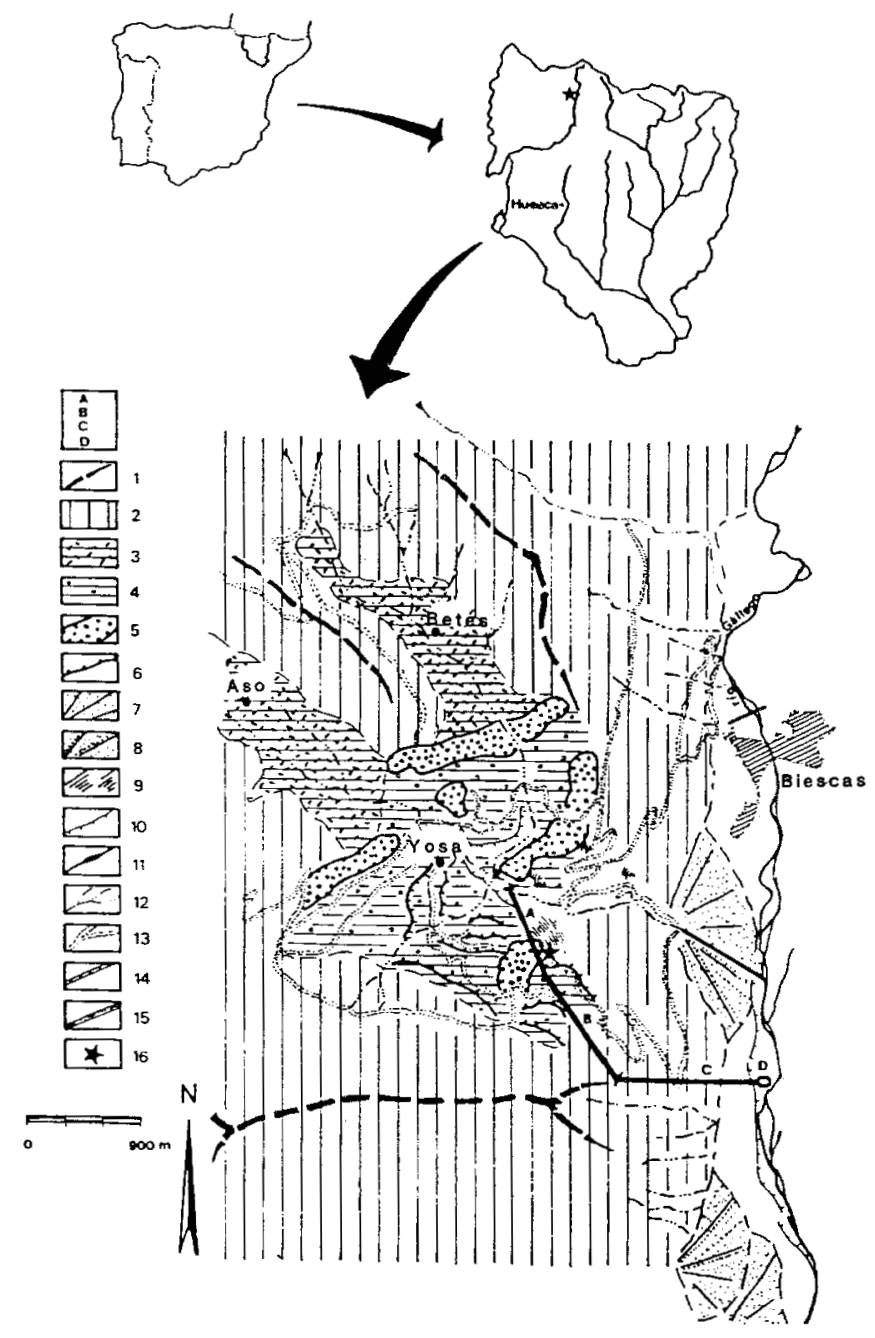

Fig. 1.- Localización del área de estudio y esquema geomorfológico del barranco de Aso. A.- Azud. B.- Canal de derivación. C.- Tubería forzada. D.- Central; 1.Interfluvios alomados sobre la facies flysch. 2.- Laderas regularizadas sobre la facies flysch. 3.- Depósitos de obturación glacial. 4.- Depósitos morrénicos derrubiados y fosilizados por depósitos de ladera. 5.- Cordones morrénicos. 6.- Escarpes de banquetas morrénicas. 7.- Conos de deyección no funcionales. 8.- Conos de deyección funcionales. 9.- Cárcavas. 10.- Escarpes sobre acumulaciones aluviales en el valle del Gállego. 11.- Cauce del río Gállego. 12.- Barrancos. 13.- Pistas forestales y caminos. 14.- Puente sobre el río Gállego. 15.- Canal artificial en el tramo bajo del barranco de Aso. 16.- Localización de las Señoritas de Arás. (Fuente: GARCIA RUIZ y PUIGDEFABREGAS 1982, y elaboración propia). 
Fundamentalmente el río Gállego, de trazado norte-sur, y sus principales afluentes en la Ribera de Biescas, barrancos de Aso por la derecha, y Sía, Espierre y Oliván por la izquierda, han sido capaces de romper la monotonía del relieve sobre flysch, abriendo pasillos en sentido transversal al del valle principal, esto es, esteoeste.

Sobre este marco morfológico se instala una cubierta vegetal, intensamente antropizada, densa en las laderas orientadas al Norte, con bosques de Pinus sylvestris, pino rojo, sobre un sotobosque de boj y aliagas, y abierta en las laderas de solana con matorral dominante de aliagas, gramíneas... en cultivos abandonados sobre suelos pedregosos con una escasa capacidad de almacenamiento hídrico.

El clima del Sobremonte es de tipo submediterráneo, con oscilaciones térmicas fuertes, un mínimo pluviométrico estival y dos máximos equinocciales, registrando intensidades de $90 \mathrm{~mm}$./24 horas en Biescas (existen máximas intensidades de 37 $\mathrm{mm}$./hora en Jaca), que suelen ocurrir a finales de verano o en otoño; el $40 \%$ de la precipitación recogida en Aso $(\mathbf{1 2 6 4} \mathrm{m}$.) es de carácter nival, teniendo en cuenta que el manto de nieve perdura en las laderas orientadas hacia el Norte, mientras que en las solanas provoca manifestaciones periglaciales sobre el suelo desnudo.

El sistema morfogenético, derivado de la interrelación de elementos tales como topografía, vegetación, orientación, clima..., aparece decididamente matizado por un factor que en el Sobremonte se convierte en fundamental, la aparición de conjuntos morrénicos sobre el flysch eoceno, y los mecanismos derivados de la morfogénesis fluvial postglacial.

\section{EL MODELADO GLACIAL DEL GALLEGO}

El modelado glacial en la Ribera de Biescas presenta su principal manifestación en un arco morrénico frontal localizado en Senegüié (832 m.), que parece enlazar con el nivel fluvial del Gállego situado a $+15-20 \mathrm{~m}$; a esta acumulación habría que añadir algunos restos de morrenas también frontales, prácticamente desaparecidas por la actividad de las aguas de origen fluvio-glacial.

El glaciar del Gállego, alimentado por los altos relieves del Valle de Tena, en su salida de las Sierras Interiores por Santa Elena, alcanzaba un espesor en torno a los $600 \mathrm{~m}$., mientras que en la cubeta terminal de Biescas se adelgaza hasta los $400 \mathrm{~m}$., presentando una anchura de $2 \mathrm{~km}$. 
Las laderas que flanquean al Gállego en la Ribera muestran una morfología claramente en artesa, con un desnivel sobre el fondo de valle en torno a los 200-300 m. y unas pendientes con valores entre el 50-60\%, remodeladas por movimientos en masa y acumulaciones postglaciales.

Pero el glaciarismo del Gállego en este sector no se limita espacialmente al valle principal, reconociéndose un modelado glacial en los valles drenados en la actualidad por los barrancos del Puerto, Sía, Espierre o el mismo de Aso.

El glaciar del Gállego desbordó, durante el máximo glacial, los límites morfológicos del actual valle fluvial generando pequeñas lenguas laterales y construyendo morrenas de obturación en la fase de expansión.

En el barranco de Aso se localiza, precisamente, un modelado complejo (Fig.1) integrado por cordones laterales, arcos y banquetas morrénicas a diferentes niveles señalando, tal y como reconoce BARRERE (1966), la etapa de adelgazamiento del glaciar.

La morfología actual es de "morrenas" derrubiadas, formas poco netas donde, además de los depósitos glaciales -tills basales y supraglaciales-, ha tenido lugar una acumulación de materiales de diferente procedencia, coluvial, fluvial e incluso lacustre a partir de la formación de cubetas de obturación.

Este carácter poligénico de los materiales explica su variedad facial y su heterometría; grandes bloques de cuarcitas, pizarras y en menor medida de granito, envueltos con una matriz arcillosa, que incluye clastos calcáreos y areniscosos del flysch eoceno. En los tramos superficiales de algunos depósitos se observa un buzamiento de los clastos hacia el Norte y Noroeste, resultado de la expansión del cono de un torrente supraglacial que ha fosilizado los sedimentos acumulados en el valle obturado por los cordones morrénicos.

La edad de los restos morrénicos tanto en el valle del Gállego como en el vecino valle del Aragón, es difícil de establecer. Trabajos de detalle, realizados en el alto Noguera Ribagorzana, (VILAPLANA, 1983) atribuyen a los depósitos correspondientes a la morrena frontal del Gállego en Senegüé una edad coincidente con la primera fase del Würm, mientras que MARTI BONO (1975) señalaba una edad anterior, Pre-Würmiense, para las morrenas más importantes, a partir de criterios relacionados con el grado de alteración de los materiales que las constituyen; MARTI BONO, SERRAT y GONZALEZ (1977) reconocen una fase de estacionamiento del máximo glacial, durante la cual se construyeron los arcos frontales y laterales más claros, como Senegüé para el Gállego. SERRANO (1991) data concretamente el arco 
externo morrénico del Sobremonte como máximo glacial, y BORDONAU (1992) señala para el glaciarismo pirenaico una fase de estabilización post-máximo que explicaría la existencia de ambientes glacio-lacustres yuxtaglaciales, como resultado de la obstrucción del drenaje de valles tributarios pirenaicos; por último, CHUECA y LAMPRE (1994) hacen una alusión específica a los depósitos fluvioglaciales y glaciolacustres del barranco de Arás, incluyéndolos en la fase de máximo glacial pirenaica en torno a los 50.000-45.000 BP.

\section{SIGNIFICACION GEOMORFOLOGICA DE LAS SEÑORITAS DE ARAS}

Sobre los depósitos poligénicos sustentados por la acumulación transversal inferior del barranco de Aso se han modelado magníficos ejemplos de chimeneas de las hadas que, como se ha indicado, reciben en la comarca el apelativo de "Señoritas de Arás".

Las "señoritas de Arás" se localizan en la orilla derecha del Barranco de Aso, a unos 1050 m.de altitud. Son dos chimeneas de $15-20 \mathrm{~m}$. de altura formadas por un cuello integrado por finos arcillosos y cantos angulosos de cuarcitas y pizarras que sustenta dos bloques de cuarcita, de dimensiones métricas.

Estas formas han sido esculpidas por la erosión hídrica sobre un depósito fundamentalmente morrénico, que constituye el arco inferior del complejo glacial del Sobremonte. Se trata de un arco derrubiado y fosilizado, tanto por material procedente de la ladera flyschoide como por un cono yuxtaglacial, incidido por la activa erosión lineal remontante del barranco de Aso. Por lo tanto, la acumulación representa una formación fácilmente erosionable, si tenemos en cuenta el pronunciado perfil longitudinal del barranco, la fuerte pendiente de la ladera en la que se insertan $(>50 \%)$, la escasa diagénesis que presenta el material y otros factores relacionados con la cubierta vegetal y edáfica o con las características climáticas actuales de la zona, recogidas someramente en anteriores epígrafes.

La inestabilidad de las laderas constituidas por acumulaciones glaciales, ya ha sido puesta de manifiesto en trabajos como el de GARCIA RUIZ y PUIGDEFABREGAS (1982) en el valle del Gállego

El resultado de la erosión hídrica y la caída de rocas es el vaciado parcial del depósito, a partir de la acción de las aguas corrientes -erosión lineal del barranco de Aso y acarcavamiento a cargo de surcos vivos instalados sobre la acumulación detrítica-; esta actividad ha modelado las chimeneas, que se encuentran en un 
avanzado estado de evolución, si bien parecen esbozarse nuevas morfologías sobre el escarpe del depósito que mira hacia el Norte y Nordeste.

No se conocen tasas de erosión y arrastre de material referidas a las cárcavas tributarias del barranco de Aso; no obstante se han revisado trabajos realizados en otros ámbitos espaciales donde pudieran confluir algunos elementos naturales comparables a los localizados en el entorno de las "Señoritas de Arás".

GALLART, CLOTET y BALASCH (1988) en la cuenca de Vallcebre (valle del Llobregat, Pirineo Oriental) a $1119 \mathrm{~m}$., sobre margas cretácicas, reconocen tasas de erosión que oscilan entre $14 \mathrm{~kg} . / \mathrm{m}^{2}$ y $23 \mathrm{~kg} . / \mathrm{m}^{2}$, cuantificando el peso del sedimento evacuado, si bien corresponden a un período particularmente húmedo. La erosión, a partir de estos datos, supone un retroceso de $9 \mathrm{~mm}$./ año en las laderas acarcavadas, teniendo en cuenta siempre que estas tasas podrían entenderse como representativas de cárcavas sobre margas en la montaña mediterránea.

Estos autores señalan que los acarcavamientos recientes estarían en relación con precipitaciones tormentosas esporádicas y los efectos de la actividad humana, deforestando las laderas y favoreciendo, por lo tanto, los movimientos en masa.

No se pueden asumir estas velocidades de retroceso sin conocer otras características paisajísticas tales como pendiente, cubierta vegetal..., que condicionan las tasas de erosión. No obstante, sí puede afirmarse la precariedad de las "Señoritas de Arás" bajo condiciones estrictamente naturales.

\section{SIGNIFICACION ESTETICO-PAISAJISTICA DE LAS SEÑORITAS DE ARAS}

Los aspectos perceptivo-sensoriales del paisaje, como elemento estético y expresión de una armónica relación de elementos naturales y culturales, se consideran como una variable independiente en el estudio del medio natural y un recurso y bien patrimonial que es preciso usar y gestionar adecuadamente. Las Señoritas de Arás desde esta perspectiva toman una significación inédita; constituyen un hito de interés muy singular dentro del paisaje de la Ribera de Biescas, una continua fuente de sensaciones, un recurso de bienestar y un documento por descifrar.

Las Señoritas de Arás contienen y emiten una serie de signos propios a través de los que comunican su identidad, a la vez que impresionan estéticamente. La estimación diferenciada y objetivable de determinados elementos y factores visuales y su composición en la escena, como transmisores esenciales de información paisajística y 
estimuladores de sensaciones estéticas, puede ayudar a codificar y valorar el significado como recurso del paisaje estudiado.

Desde cualquier lugar de observación, por distante que esté, los bloques que coronan las chimeneas constituyen sendos puntos de energía en el campo visual; su significado es esencialmente señalizador y marcador en el espacio y llaman poderosamente la atención. Las líneas verticales y los volúmenes cilíndricos de las columnas, enmarcados en la mancha rojiza de la tierra desnuda, tienen asimismo enorme fuerza, especialmente en un paisaje predominantemente verdoso donde los elementos verticales y de geometrías simples no abundan. Estos elementos básicos cobran mayor significación cuando se consideran sus características de tamaño, textura, valor de luminosidad, dirección, forma y color. El tamaño de las Señoritas de Arás, expresión de las diferencias de magnitud y sus relaciones, es relativamente pequeño en un paisaje de grandes dimensiones, sin embargo impresiona cuando la referencia es el hombre mismo. La textura, referida a las variaciones o irregularidades de una superficie, la luminosidad y el color establecen marcadas diferencias entre el espacio de las laderas próximas cubiertas de bosque y el desgarro erosivo en el que se encuentran las Señoritas. La forma cilíndrica y el extraplomo del bloque superior, unido a la dirección vertical de las chimeneas en agudo ángulo con la ladera fuertemente inclinada, introducen una fuerte tensión en la escena.

La regularidad, sencillez formal y predictibilidad en la evolución de las Señoritas contrasta vivamente con la irregularidad, profusión y espontaneidad de las cárcavas y de las distribuciones de los matorrales espontáneos. El dinamismo determinado por las líneas diagonales convergentes o secantes del valle en ' $V$ ' determina un movimiento en la imagen bien diferente al estatismo que provocan las chimeneas verticales. La neutralidad y continuidad de los bosques y las praderas de las colinas monótonas se rompe con el acento de las dos columnas aisladas que interrumpen con un trazo luminoso y vertical la mancha homogénea de color y la línea ondulante del horizonte. La claridad de contornos, finalmente, difiere radicalmente de los límites difusos de la cubierta vegetal.

El movimiento del caminante, necesariamente lento por las difíciles condiciones de accesibilidad, introduce una nueva variable en la percepción del paisaje: secuencia temporal; el ritmo pausado y la variación paulatina del punto de observación potencia vivamente la vibración estética.

Estos son algunos de los muchos factores, determinados por las infinitas combinaciones de los elementos y las variables visuales, con los que se pueden construir las formulaciones visuales del paisaje; no se agota aquí el catálogo, ni mucho menos, pero son probablemente suficientes para señalar el potencial estético de las Señoritas de Arás. 


\section{PROYECTO DE 'MINICENTRAL' DE ARAS}

\section{Características del Proyecto}

Antes de analizar las implicaciones ambientales de este proyecto sobre las "Señoritas de Arás", conviene definir qué se entiende por "minicentral". Según la actual legislación española, una minicentral hidroeléctrica es aquélla que necesita un azud de menos de $100.000 \mathrm{~m} 3$ de capacidad de embalse y una presa inferior a los $15 \mathrm{~m}$. de altura (Ver Real Decreto 1131/88, de 30 de septiembre, "Reglamento para la ejecución del R.D.L. 1302/86, de 28 de junio, de Evaluación de Impacto Ambiental").

La minicentral proyecta detraer un caudal del Barranco de Aso -también denominado Barranco de Arás-, tributario del Gállego, por medio de un azud que captaría sus aguas y las derivaría por la margen derecha a través de un canal hasta dejarlas caer por una tubería de gran salto.

Esta somera descripción del proyecto ya permite entrever las distintas infraestructuras que configurarían el conjunto del aprovechamiento hidroeléctrico. Además de la central propiamente dicha, existen varios elementos complementarios e interrelacionados, cada uno de los cuales debe ser analizado por separado para detectar sus previsibles impactos medioambientales (Fig. 1). Sus características principales se detallan a continuación ${ }^{1}$ :

A. Azud. Proyectado en hormigón, su base se situaría a $1.085 \mathrm{~m}$. de altitud, aproximadamente $400 \mathrm{~m}$. aguas abajo de la localidad de Yosa de Sobremonte. La longitud del labio sería de $20 \mathrm{~m}$. y su altura, de 10 .

B. Canal de derivación. Se construiría a media ladera, con una sección interior de $0,90 \times 0,80 \mathrm{~m}$; la estructura se proyecta de hormigón armado provisto de tapa y estaría inserto en una plataforma de $4 \mathrm{~m}$. de anchura, con una longitud de $3.000 \mathrm{~m}$. El desnivel que salvaría desde la cabecera hasta el final sería de $4 \mathrm{~m}$.

C. Cámara de carga.

D. Tubería forzada. Construida en acero al carbono, iría enterrada. Su diámetro interior sería de $0,6 \mathrm{~m}$. y discurriría a lo largo de $750 \mathrm{~m}$., superando un salto bruto de $245 \mathrm{~m}$.

\footnotetext{
1 Fuente: "Proyecto de concesión de minicentral hidroeléctrica. Aso-Huesca". (No de solicitud en la Comisaría de Aguas de la Confederación Hidrográfica del Ebro: 87-M-97).
} 
E. Central eléctrica. Con una potencia instalada de 2 × $600 \mathrm{kw}$.

F. Canal de restitución. Iría excavado en tierra. El resto de las características no están especificadas.

G. Línea de evacuación de la energía. Aérea, de 20 kw., y con enganche en una línea que recorre actualmente el fondo del valle del Gállego, a $1 \mathrm{~km}$. de distancia.

H. Accesos a estas infraestructuras. Sin especificar.

En general, se puede afirmar que el proyecto de ingeniería peca de inconcreto, no especifica suficientemente cuestiones de gran importancia tanto desde el punto de vista técnico como medioambiental, y en ocasiones presenta fallos evidentes. Estas carencias, algunas de las cuales se indican seguidamente -aquéllas que afectan a elementos de importancia ambiental- son puestas en varios casos de manifiesto por la propia Diputación General de Aragón ${ }^{2}$.

- El canal de derivación tendría, según los planos, $1.250 \mathrm{~m}$. de longitud, y no 3.000 como indica el proyecto. Se plantea excavado a media ladera, pero no define la inclinación de los taludes.

- No se citan las características constructivas de la tubería forzada; sí se indica que las pistas de acceso para su construcción -que no detallan su trazado- no superarán la pendiente del 6\%, lo que supondría la ocupación de una amplísima franja en una ladera de gran visibilidad, situada en el contacto con el fondo plano del valle del Gállego, junto a un camping y en una zona de evidente vocación y actividad actual turística.

- La reposición de los caudales turbinados al cauce natural, mediante el canal de restitución, no se especifica suficientemente, pero sí parece que se realizaría como simple excavación en tierra, sin ninguna medida de protección contra la fuerza erosiva de las aguas.

- La superficie afectada por la denudación vegetal que provocarían las distintas infraestructuras es, según el proyecto técnico, de sólo $12.000 \mathrm{~m}^{2}$. Sin embargo, la D.G.A. calcula por su parte una cantidad casi diez veces mayor, de $112.500 \mathrm{~m}^{2}$. Conviene recordar aquí los impactos que esta deforestación provocaría, principalmente de tipo paisajístico y erosivo, por la acusada inestabilidad del

2 En su estudio titulado "Evaluación del Estudio de Impacto Ambiental y Proyecto Técnico de la Minicentral Hidroeléctrica de Aso (Huesca)". 
sustrato litológico de las zonas afectadas -depósitos morrénicos, coluviales y flysch-.

- Se prevé un volumen global de materiales a excavar cifrado en $38.838 \mathrm{~m}^{3}$. Sus repercusiones paisajísticas, geomorfológicas y de destrucción de la cubierta vegetal serían de considerable magnitud.

\section{ANALISIS DEL ESTUDIO DE IMPACTO AMBIENTAL}

Junto al Proyecto Técnico del que se acaba de realizar un breve resumen, la empresa peticionaria ha presentado ante los organismos administrativos competentes en la materia un Estudio de Impacto Ambiental titulado "Proyecto de evaluación de impacto ambiental de la minicentral hidroeléctrica de Aso". Antes de hacer referencia a algunos puntos del mismo, es necesario indicar que su título es totalmente erróneo, ya que en ningún caso se trata de una Evaluación de Impacto Ambiental -que supone un proceso administrativo conducente a la Declaración de Impacto Ambiental, del cual el Estudio de I.A. no es más que una parte, eso sí, muy importante- sino simplemente un Estudio de Impacto Ambiental. Es decir, se confunden de partida conceptos netamente diferentes que quedan, además, perfectamente precisados en la legislación española (R.D.L. 1302/1986 y R.D. 1131/1988) y aragonesa (Decreto 45/1994) al respecto.

Un Estudio de Impacto Ambiental (E.I.A.) debe analizar las alteraciones que las infraestructuras proyectadas causarían sobre los distintos componentes medioambientales -medio físico y medio socioeconómico-, de ahí que el listado de elementos a considerar resulte bastante prolijo, tal como indica el M.O.P.U. (1989). En este artículo nos vamos a referir exclusivamente al ámbito de las "Señoritas de Arás", sin entrar a valorar los impactos causados sobre otros sectores incluidos en el proyecto de aprovechamiento hidroeléctrico.

Las alteraciones que experimentarían estas formaciones afectarían principalmente al sustrato litológico, a la morfología y al paisaje.

El E.I.A. al que hacemos referencia presenta un mapa geológico a E. 1:50.000 que no es más que una traslación del mapa de síntesis a E. 1:200.000 del I.G.M.E. (hoja 23, Huesca); describe someramente la geología general del Pirineo Axil, que nada tiene que ver con el sector de flysch y de depósitos detríticos donde se ubica el área afectada. Además, realiza una identificación litológica totalmente incorrecta, pues señala la presencia de materiales continentales del Oligoceno, no existentes en la zona, a la vez que ignora el flysch eoceno, que es la formación más importante. 
La carencia de datos estructurales y estratigráficos, unida a la incorrecta descripción litológica, imposibilitan la obtención de las necesarias consideraciones geotécnicas.

La descripción geomorfológica es totalmente genérica y se ignoran datos fundamentales, como la estabilidad de laderas y los procesos morfogenéticos. La existencia de las dames coiffées, el elemento morfológico más llamativo de la zona, se obvia totalmente.

Los datos climáticos, necesarios para estudiar los procesos que afectan a la morfogénesis, se obtienen a partir del ajuste de los valores de la estación meteorológica de Panticosa, elección extraña existiendo mucho más cerca, y con comportamiento climático más similar al del barranco de Aso, las estaciones de Biescas y de Aso de Sobremonte (CREUS, 1983; CUADRAT, 1981).

Respecto al paisaje, el E.I.A. señala su poca relevancia y que la visibilidad de las obras será escasa. Sin embargo, olvida citar la existencia de las "Señoritas de Arás", elementos singulares únicos, recogidos además por el I.C.O.N.A. y por el M.O.P.U. en su "Inventario abierto de espacios naturales de protección especial" (I.C.O.N.A. y M.O.P.U., 1978). La afirmación de que la visibilidad será escasa es muy discutible, pues el sector que nos ocupa es perfectamente visible desde bastantes puntos del valle del Gállego e incluso desde núcleos habitados, situados fuera del propio valle (Gavín, entre otros).

La calidad medioambiental (paisajística, geomorfológica, científica y educativa) de estas dames coiffées vuelve a ser puesta de manifiesto en el que se puede considerar como el estudio más ambicioso y reciente llevado a cabo en Aragón en materia de propuesta de protección de espacios naturales, la RENPA (Red de Espacios Naturales Protegibles de Aragón), trabajo encargado por la Dirección General de Ordenación Rural del Departamento de Agricultura, Ganadería y Montes de la Diputación General de Aragón. En sus diferentes versiones (D.G.A, 1989; D.G.A., 1991), el espacio denominado "Señoritas de Arás" aparece catalogado con la figura de protección de Monumento Natural, una de las que permite la Ley estatal 4/1989, de Conservación de los Espacios Naturales y de la Flora y Fauna Silvestres.

\section{VALORACION CRITICA}

Tras el análisis somero de las características técnicas del proyecto y de su E.I.A., la impresión recibida es la de falta de la necesaria concreción y seriedad de los mismos, algo que desgraciadamente no es exclusivo de este caso sino que resulta 
bastante habitual en proyectos de este tipo. Los organismos que tienen competencia para aprobarlo o rechazarlo son la Diputación General de Aragón y la Confederación Hidrográfica del Ebro. Por este motivo, la primera ha realizado un informe, ya citado en páginas anteriores, en el que pone de manifiesto las carencias detectadas y propone diferentes recomendaciones.

En el caso de las alteraciones que sufririan las "Señoritas de Arás" y su entorno, las conclusiones son bastante claras y, parcialmente, se exponen a continuación:

- El canal de derivación y el/los camino/s de acceso entre el azud y la cámara de carga, son los elementos que afectan a las Dames Coiffées y a los depósitos morrénicos sobre los que se han elaborado.

- Este canal se plantea excavado a media ladera y sobre él se construiría el camino. No se indica la inclinación de sus taludes y terraplenes, algo totalmente necesario si se tiene en cuenta que el perfil de equilibrio de la ladera en algunos tramos no supera la pendiente del $50 \%$.

- Los fenómenos erosivos, además de por los movimientos de tierras, se verán magnificados por la destrucción de la cubierta vegetal, consistente en ejemplares aislados de pinos y sauces sobre el depósito morrénico y en un bosque denso de pinar silvestre con sotobosque y claros de boj en los niveles superiores de la ladera, sobre el flysch, que previsiblemente se verá afectado por una intensa erosión remontante, en la actualidad ya muy activa.

- La repoblación propuesta en el E.I.A., con la finalidad de estabilizar los taludes y de ocultar las infraestructuras, es muy problemática en todo este sector morrénico, por la magnitud de los deslizamientos que se generarán.

- El canal y el camino discurrirán en un tramo de unos $600 \mathrm{~m}$. por la morrena, a muy pocos metros por encima de las "Señoritas de Arás". Tal como reconoce el informe de la D.G.A., el frágil equilibrio en que éstas se encuentran ocasionará su desaparición "con toda seguridad, por las simples vibraciones de la maquinaria".

Tras realizar estas precisiones -y otras, que por afectar a otros sectores y a otros elementos medioambientales no incluimos aquí-, el informe de la D.G.A. termina con una serie de recomendaciones, de las que citamos textualmente el primer párrafo:

"La recomendación inicial tras la valoración de los posibles impactos es la de considerar compatible la realización del Proyecto de minicentral de Aso". 
Esta consideración de impacto compatible sólo se mantiene si se aprueban determinadas medidas correctoras tendentes a minimizar los impactos negativos, sin las cuales "no debería ser aceptado el Proyecto". De este paquete de medidas extraemos aquéllas que más nos interesan:

- "Presentar un proyecto constructivo de la totalidad de los caminos, tanto provisionales como definitivos, a E. 1:1.000 (...), en donde se pueda analizar exactamente la amplitud de la excavación y de los terraplenes a realizar (...) y las medidas correctoras de fijación de laderas y revegetación propuestas (...)".

- "Diseñar y aplicar un proyecto de protección y consolidación, durante la fase constructiva, de las chimeneas de las hadas".

La primera nos parece totalmente necesaria y coincidimos con la D.G.A. en que "sin este documento no es posible evaluar con precisión la magnitud del impacto". Pero, inmediatamente, surge la duda: ¿cómo es posible, entonces, que a pesar de esta carencia se haya valorado el impacto?.

La segunda de las medidas es, en nuestra opinión, perfectamente inútil, carente de la menor efectividad. No hay que olvidar que, como el informe reconoce y ya se ha dicho, las simples vibraciones de la maquinaria harían dasaparecer a las "Señoritas de Arás".

Aún suponiendo que las medidas de protección y consolidación fuesen efectivas, las "Señoritas" perderían su actual estatus de formaciones naturales, quedando como una especie de monumento restaurado. Además, su entorno resultaría totalmente alterado por las infraestructuras proyectadas, de forma que el valor ambiental del conjunto -reconocido por el I.C.O.N.A., el M.O.P.U. y la D.G.A., como ya se ha indicado y por citar sólo a los organismos oficiales- disminuiría notablemente.

Conviene conocer con exactitud qué significado tiene el concepto de impacto compatible. El R.D. 1131/1988 establece con total precisión una gama de calificaciones de impactos ambientales que, en el caso de los negativos, abarca desde el compatible hasta el crítico. Sus significados, copiados textualmente de este R.D. (anexo 1: Conceptos Técnicos) son los siguientes:

- "Impacto ambiental compatible: aquél cuya recuperación es inmediata tras el cese de la actividad y no precisa prácticas protectoras o correctoras.

- Impacto ambiental moderado: aquél cuya recuperación no precisa prácticas protectoras o correctoras intensivas, y en el que la consecución de las condiciones ambientales iniciales requiere cierto tiempo. 
- Impacto ambiental severo: aquél en el que la recuperación de las condiciones del medio exige la adecuación de medidas protectoras o correctoras y en el que, aún con esas medidas, aquella recuperación precisa un período de tiempo dilatado.

- Impacto ambiental crítico: aquél cuya magnitud es superior al umbral aceptable. Con él se produce una pérdida permanente de la calidad de las condiciones ambientales, sin posible recuperación, incluso con la adopción de medidas protectoras o correctoras".

En nuestra opinión, no queda lugar a la duda de que la calificación otorgada aún provisionalmente- por el informe de la D.G.A., de impacto compatible, no se ajusta a la realidad. En absoluto se produce una recuperación inmediata tras el cese de la actividad. Más bien estimamos que la recuperación de las "Señoritas de Arás" es imposible a la escala humana del tiempo. Si a esto sumamos el gran valor ambiental derivado de su singularidad, podemos considerar el impacto como crítico, por lo que el proyecto de aprovechamiento hidroeléctrico nos parece totalmente inviable.

Ateniéndonos a los trabajos de planificación territorial y a la normativa más reciente de la D.G.A, además de a sus proyectos legislativos, parece muy improbable que este proyecto llegue a aprobarse. En primer lugar, el Plan Especial de Protección del Pirineo (D.G.A., 1993), que desarrolla las Directrices Generales de Ordenación Territorial del Pirineo Aragonés (no aprobadas en la fecha de presentación de este artículo -septiembre de 1994-, por lo que no resulta vinculante desde el punto de vista legal), propone la declaración de espacio protegido de las "Señoritas de Arás". En concordancia con el artículo 19 de la Ley 11/1992, de Ordenación del Territorio de Aragón, se prevé para este espacio una estrategia protectora activa (si se llega a calificar como Monumento Natural, algo en lo que coinciden las distintas propuestas de la D.G.A. (D.G.A., 1989, 1991 y 1993). Bajo esta protección, la autorización de los diferentes usos del suelo "queda vinculada, dependiente y subordinada a la protección del medio. Por lo tanto no podrán realizarse obras ni actividades que perjudiquen al medio natural (...)".

Otras normas legales aragonesas referidas a medidas de protección urbanística, afectan plenamente al espacio que nos ocupa. Así ocurre con los decretos 85/1990 y 14/1991 de la Diputación General de Aragón, de medidas urgentes de protección urbanística en Aragón y sobre protección urbanística del territorio propuesto para la posible realización de los Juegos Olímpicos de Invierno en Jaca (Huesca), respectivamente. Por otro lado, el Proyecto de Ley de Espacios Naturales Protegidos de Aragón, actualmente en tramitación en las Cortes de Aragón, incluye en la relación de espacios propuestos a las "Señoritas de Arás" (CANCER, 1994). 


\section{CONCLUSIONES}

Las "Señoritas de Arás" constituyen un magnífico ejemplo de las morfologías cuaternarias que manifiestan un acusado valor ambiental, estético y cultural.

La dilatada evolución postglacial de los arcos morrénicos del Sobremonte ha abarcado mecanismos de escorrentía superficial, concentrada y difusa, procesos gravitatorios, actividad eólica, movimientos en masa, intervenciones antrópicas... que han modelado formas singulares, no bajo un criterio exclusivamente geomorfológico, sino también paisajístico.

La singularidad de estas "dames coiffées" justifica sobradamente el dictado de medidas tendentes a su protección efectiva, al igual que para su entorno inmediato y, en consecuencia, proyectos que suponen una drástica alteración medioambiental, como el aquí presentado, deberían desecharse, para lo cual habría que hacer uso de la normativa legal actualmente en vigor.

\section{BIBLIOGRAFIA}

ALONSO, R. y PELLICER, F. (1992): El paisaje entre la ciencia y el arte, Universidad Hispanoamericana de Sta. $\mathrm{M}^{\mathrm{a}}$. de la Rábida.

BARRERE, P. (1966): "La morphologie quaternaire dans la règion de Biescas et de Sabiñanigo (Haut Aragon)". Bulletin de l'Asociation Francaise pour l'Etude du Quaternaire , 2, 83-88.

BORDONAU i IBERN, J. (1992): Els complexos glacio-lalcustres relacionats amb el darrer cicle glacial als Pirineus. Geoforma Ediciones, $251 \mathrm{p}$.

CANCER, L. (1994). Ecogeografía de los paisajes del Alto Gállego. Bases físicas para su ordenación territorial. Dpto. de Geografía y Ordenación del Territorio, Universidad de Zaragoza. (Tesis Doctoral inédita).

CREUS, J. (1983). El clima del Alto Aragón occidental. Monografías del Instituto de Estudios Pirenaicos, 109. C.S.I.C., Jaca.

CUADRAT, J.M. (1981). El clima del Pirineo Central. Ensayo de aplicación al turismo de montaña. Dpto. de Geografía, Universidad de Zaragoza. (Tesis Doctoral inédita).

COROMINAS, J. (1990): "Influencia del glaciarismo cuaternario en la estabilidad de las laderas del valle del Valira d'Orient (Andorra)". Actas de la I Reunión Nacional de Geomorfología, 521-532. 
CHUECA,J. y LAMPRE, F.,(1994): "Los glaciares altoaragoneses", Cuadernos Altoaragoneses de Trabajo, 21, 22 p. Instituto de Estudios Altoaragoneses. Huesca.

D.G.A. (1989). Red de espacios Naturales Protegibles de la Comunidad Autónoma de Aragón (RENPA). (1a. versión: marzo de 1989). Dirección General de Ordenación Rural. Dpto. de Agricultura, Ganadería y Montes. (Inédito).

D.G.A. (1991). Red de espacios Naturales Protegibles de la Comunidad Autónoma de Aragón (RENPA). (Versión de septiembre de 1991). Dirección General de Ordenación Rural. Dpto. de Agricultura, Ganadería y Montes. (Inédito).

D.G.A. (1993). Plan Especial de Protección del Pirineo. Dirección General de Ordenación del Territorio y Urbanismo.Dpto. de Ordenación del Territorio, Obras Públicas y Transportes. Zaragoza.

FONTBOTE, J.M. (1948): "La Ribera de Biescas". Pirineos, año 4, 7, 40-84.

GALLART, F., CLOTET, N. y BALASCH, C (1988): " Medium-term Erosion Rates in a Small Scarcely Vegetated Catchment in the Pyrenees". Catena Suppl. 13. 37-49.

GARCIA RUIZ, J.M. (1989): Sallent. Mapa geomorfológico Geoforma Ediciones

GARCIA RUIZ, J.M. y PUIGDEFABREGAS, J. (1982) : "Formas de erosión en el Flysch eoceno surpirenaico". Cuadernos de Investigación Geográfica, t. VIII, 83-126.

HARVEY, A.M. (1987): "Seasonality of processes on eroding gullies: a twelve-year record of erosion rates", Proccessus et messure de l'érosion, 439-454. C.N.R.S.

I.C.O.N.A. y M.O.P.U. (1978). Inventario abierto de espacios naturales de protección especial. I.C.O.N.A. y Dirección General de Urbanismo del M.O.P.U., Madrid.

MARTI, C.E., SERRAT, D. y GONZALEZ, M.C. (1977): "Los fenómenos glaciares en la vertiente meridional de los Pirineos". Actas del $V$ Coloquio de Geografía. 68-73.

MARTI BONO, C. (1975): "Excursiones I y II. Altos Valles de los ríos Aragón y Gállego". Actas de la II Reunión Nacional del Grupo Español de Trabajo del Cuaternario, 337-344.

M.O.P.U. (1989): Guias para la elaboración de Estudios de Impacto Ambiental.. 2: Grandes Presas. Madrid.

PELLICER, F. (1992): Valoración estética del paisaje en el Camino de Santiago (tramo aragonés): Criterios de objetivación. En TORRES LUNA, M.P., PEREZ ALBERTI, A. Y LOIS GONZALEZ, R.C. (Eds.): Los Caminos de Santingo y el territorio, Congreso Internacional de Geografía, Xunta de Galicia. 79-104. 
VILAPLANA, J.M. (1983): "Quaternary Glacial Geology of Alta Ribagorça Basin (Central Southern Pyrenees)" Acta Geológica Hispánica, t. 18, 3/4, 217-223.

SERRANO, E. (1991): Geomorfología glacial de las montañas y valles de Panticosa y la Ribera de Biescas (Pirineo aragonés). Universidad Autónoma de Madrid (Tesis Doctoral inédita). $952 \mathrm{p}$. 